Supporting Information for

\title{
Room-temperature Ferroelectricity in Group-IV Metal
}

\section{Chalcogenide Nanowires}

Jun-Jie Zhang, ${ }^{1,2}$ Jie Guan, ${ }^{2}$ Shuai Dong ${ }^{2 *}$ and Boris I. Yakobson ${ }^{1,3^{*}}$

1Department of Materials Science and NanoEngineering, Rice University, Houston, Texas 77005, USA

${ }^{2}$ School of Physics, Southeast University, Nanjing 211189, China

${ }^{3}$ Department of Chemistry, Rice University, Houston, Texas 77005, USA

Email: sdong@seu.edu.cn; biy@rice.edu

\section{Computational Details}

The DFT calculations were performed using the Vienna ab initio simulation package (VASP). ${ }^{1,2}$ The projector-augmented wave (PAW) potentials with the generalized gradient approximation of Perdew-Burke-Ernzerhof (GGA-PBE) formulation are used with a cutoff energy of $500 \mathrm{eV}$. A vacuum region with 20 $\AA$ thickness is introduced to eliminate interaction between nanowires and Brillouin zone is sampled with $1 \times 1 \times 20 k$-points. The electronic contributions to the spontaneous polarization, defined as the difference in polarization between the ferroelectric ground state structure (polar structure) and the postulated high symmetry paraelectric phase (nonpolar structure), were calculated using the Berry phase approach. ${ }^{3,4}$ The phonon dispersion is calculated using the 
framework of density functional perturbation theory (DFPT), as implemented in the Quantum-ESPRESSO distribution. ${ }^{5}$

The bulk of GeS and SnS adopt the layered, black-phosphorus-like structure, where the interlayer interaction is van der Waals type. Thus to compare the energies of corresponding phases, the van der Waals corrections were employed (DFT-D2). More important, we found that the vdW correction has limit effects on value of polarization and $E \mathrm{p}$.

ab initio MD calculations were performed on $8 \times 1$ supercells, for up to 5000 fs with a 1.5 fs time resolution. The same basis sets and pseudopotentials as in the DFT calculations were employed.

\section{Phonon dispersion of 1D GeS and SnS}

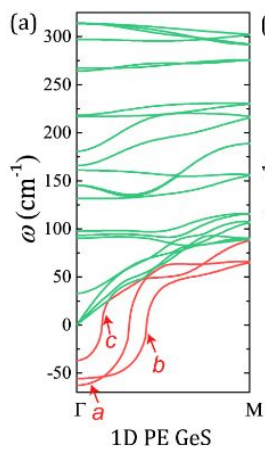

(e)

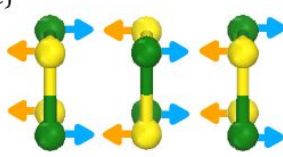

mode a

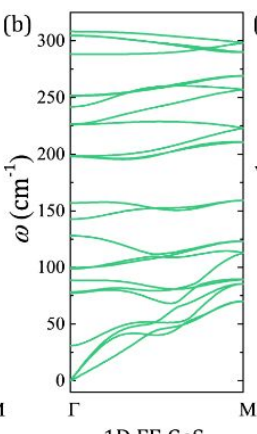

(f)

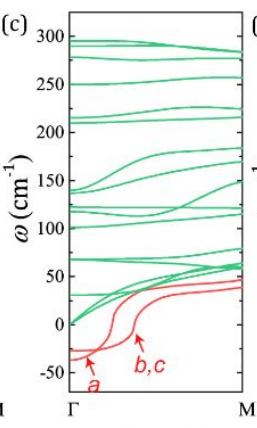

1D PE SnS (g)

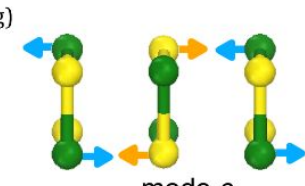

mode $c$

Figure S1. (a-d) Phonon dispersion of 1D GeS and SnS nanowire. (e-g) Imaginary frequency modes of 1D GeS and SnS nanowire. Green atom is Ge or Sn. Yellow atom is $S$.

The phonon dispersions indicate that 1D GeS and SnS PE structures are 
dynamically unstable, where three optical modes have negative frequency ( $a$ $c$ ) around $\Gamma$ points ( $b$ and $c$ are very close in SnS). The space inversion will be spontaneously removed by $a-c$ soft optical modes, resulting in the structural transition. Here, we also calculated the energies of other two new phases (resulting from $b$ and $c$ modes), which are less stable than FE structure (Table S1).

Table S1. Calculated formation energies (meV) of new phases, which are the results of negative frequencies $(a-c)$. The energy of $a$ is set as the reference.

\begin{tabular}{cccc}
\hline \hline & $a$ & $\square b$ & $\square c$ \\
\hline GeS & 0 & 32.5 & 62.4 \\
SnS & 0 & 21.2 & 32.6 \\
\hline \hline
\end{tabular}

\section{Formation energy and electronic structure of 1D GeS and SnS}

(a)

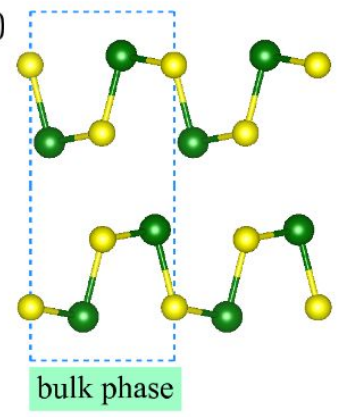

(b)

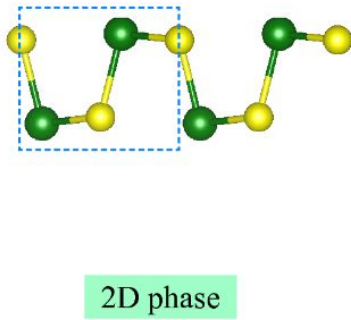

(c)

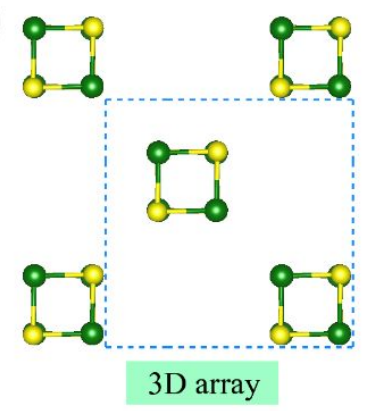

Figure S2. Sketch of (a) bulk structure, (b) monolayer structure, and (c) 3D array structure. Blue dashed line indicates selected unit cell.

Table S2. Calculated formation energies (eV) of bulk, monolayer, 1D FE, 1D PE, and 3D 
array phase. The energy of bulk structure is set as the reference.

\begin{tabular}{cccccc}
\hline \hline & Bulk & Monolayer & 1D FE & 1D PE & 3D array \\
\hline $\mathrm{GeS}$ & 0 & 0.126 & 0.231 & 0.245 & 0.186 \\
$\mathrm{SnS}$ & 0 & 0.164 & 0.290 & 0.294 & 0.232 \\
\hline \hline
\end{tabular}
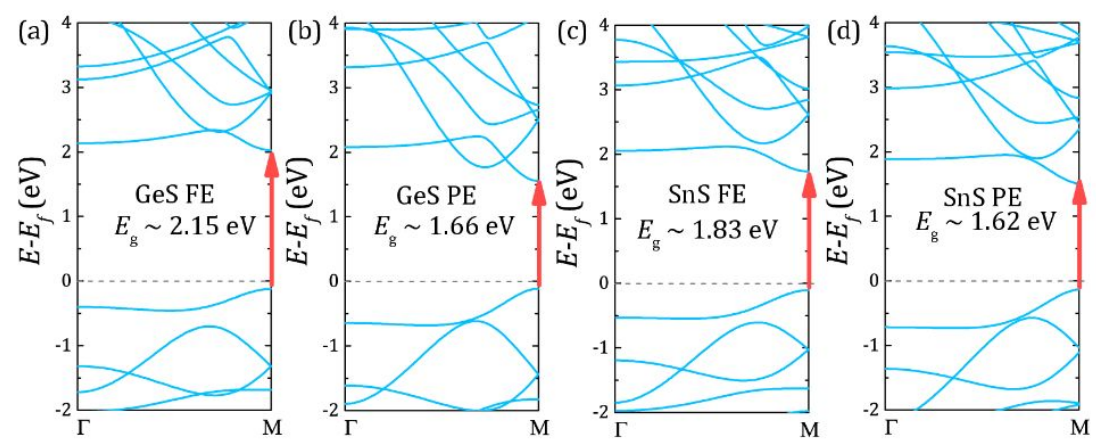

Figure S3. Electronic structure of 1D GeS and SnS nanowires.
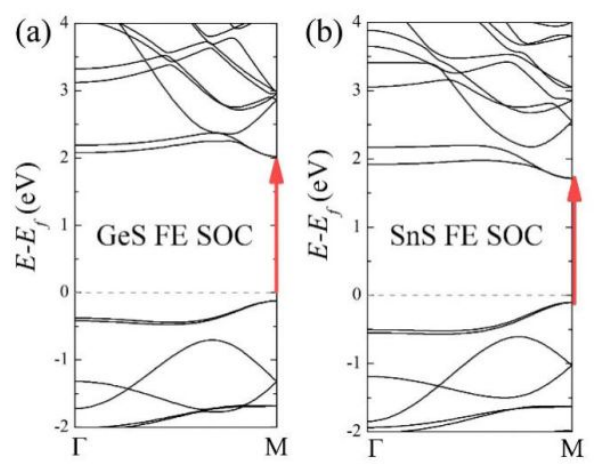

Figure S4. Band structure with SOC of ferroelectric (a) GeS and (b) SnS nanowires.

\section{Monte Carlo simulations}

The standard Markov chain Monte Carlo (MC) method with the Metropolis algorithm is employed to determine the Curie temperature. Periodic boundary conditions are adopted with lattice size $\left(1 \times 1 \times 10^{4}\right)$. In our MC simulation, the first $10^{4}$ steps (MCSs) are used for thermal equilibration, and then the additional $10^{4} \mathrm{MCSs}$ are used for measurements. The acceptance ratios of MC 
updates are controlled to be $\sim 50 \%$ at all simulated temperature, by adjusting the updating windows. The quenching process (i.e. gradually cooling from high to low $T$ ) is adopted in our $\mathrm{MC}$ simulations.

Based on Landau theory, it is expected that there is no ferroelectric transition in 1D system. Similar situation is also found in 1D isotropic magnetic system, where long-range magnetic order is hardly achieved at nonzero temperature. For example, there is no phase transition in 1D Ising system due to lack of enough neighbors. If one additional neighbor chain is included in 1D Ising model, a nonzero transition temperature is found, where interchain interactions make crucial contribution. Herein, the interaction between two nanowires is considered to achieve the Curie temperature in 1D GeS and SnS. The corresponding part in total energy can be written as $\frac{D}{2} \sum_{i j}\left(P_{i}-P_{j}\right)^{2}$. To obtain the parameter $D$, two kinds of interaction, ferroelectric (FE) and antiferroelectric (AFE) coupling between two nanowires (Figure S5), are considered here.
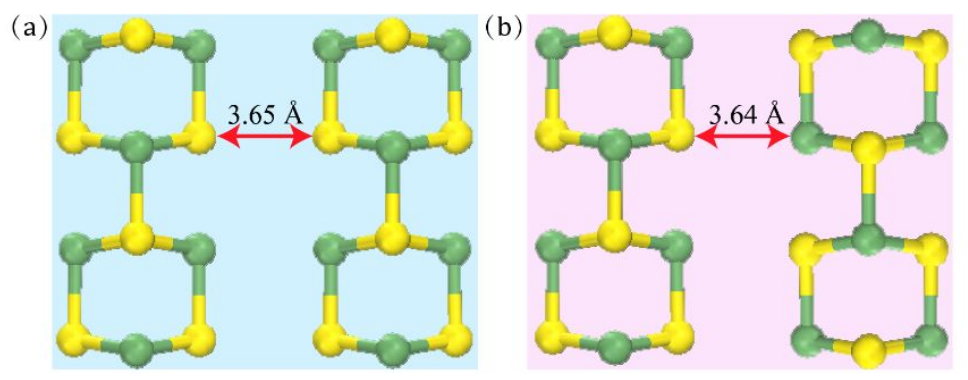

Figure S5. Sketch of dipole-dipole interaction between two nanowires.

The distances between two nanowires with FE and AFE coupling are fully relaxed, and the obtained values are around $3.6 \AA$, indicating the qusi-1D 
character. More important, we found AFE is more favorable than FE coupling (Table S3). Furthermore, to get the value of $D$ in main text, we calculated energy difference $(\Delta E)$ as a function of $P_{1}-P_{2}$. We found that they satisfy the nonlinear quadratic relationship, which is similar to the case of dipole-dipole interaction in same nanowire (Figure 3 in main text). Therefore, we obtained the values of $D$ by fitting the corresponding quadratic relationship (Table S3).

Monte Carlo (MC) simulation is performed with Eq. (1) in main text. The MC simulations give the effective $T_{\mathrm{C}}$ around $950 \mathrm{~K}$ and $690 \mathrm{~K}$ for GeS and $\mathrm{SnS}$ nanowires (Figure S6b), which is much higher than MD results. In MC simulation, $T_{\mathrm{C}}$ is based on the polar mode $a$, which is equivalent to the energy barrier ( 129 meV for GeS) between ferroelectric and paraelectric phase (the progress from double to single wall). In fact, nonpolar mode $b$ is possible to weaken $T_{\mathrm{C}}$ due to the lower energy barrier between mode $a$ and $b$ structure ( $\sim 30 \mathrm{meV}$ for $\mathrm{GeS})$, which is considered in MD methods. It means that ferroelectric phase may partly transfer to mode $b$ nonpolar structure in MD calculations.

Noted that the temperature doesn't change much within different size of MCs lattices (Figure S7), i.e. $1 \times 1 \times 10^{3}$ and $1 \times 1 \times 10^{4}$, implying the possibility to stabilize the quasi-1D ferroelectric order at finite temperature.

Table S3. Calculated energy difference between $\mathrm{FE}$ and AFE coupling ( $\left.\Delta E=E_{\mathrm{FE}}-E_{\mathrm{AFE}}\right)$ for 1D GeS and SnS. The obtained the values of $D$ are also listed. 


\begin{tabular}{ccc}
\hline & $\Delta E(\mathrm{emV})$ & $\square D$ \\
\hline Ges & 17 & -0.22 \\
Sns & 22 & -0.29 \\
\hline \hline
\end{tabular}
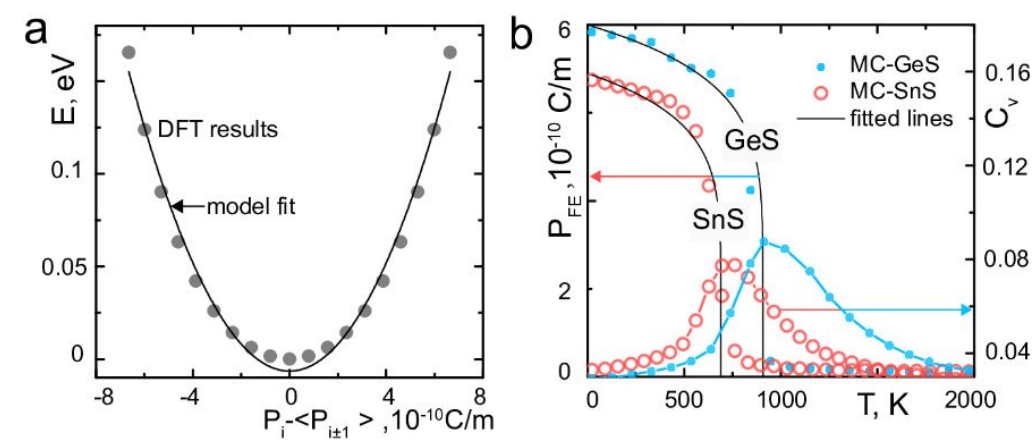

Figure S6. (a) Nearest-neighbor dipole-dipole interaction in 1D FE GeS based on meanfield theory $\mathrm{Pi}-<\mathrm{Pi} \pm 1>(10-10 \mathrm{C} / \mathrm{m})$, direct DFT data (dots) and a harmonic function fit (line). (b) FE polarization $\mathrm{P}$ and specific heat $\mathrm{C} v$ as a function of temperature $\mathrm{T}$, for a 1000 u.c. ( 200 nm) wire: MC data (dots) and fitted curves, for both GeS and SnS.
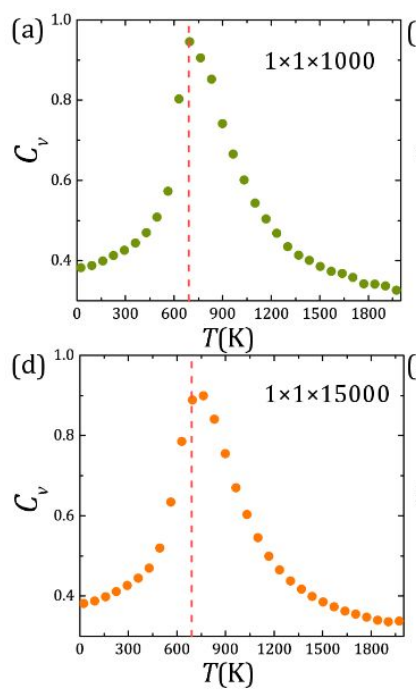
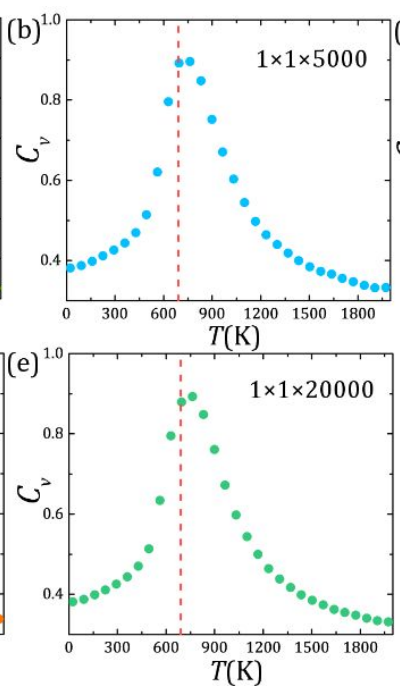

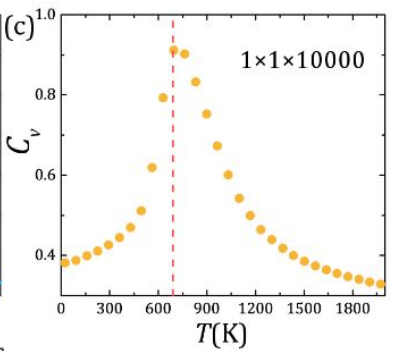

Figure S7. Obtained MCs results within various lattice. Specific heat $\left(C_{v}\right)$ is defined as 
$\partial E / \partial T$ is a function of temperature. ${ }^{6,7}$
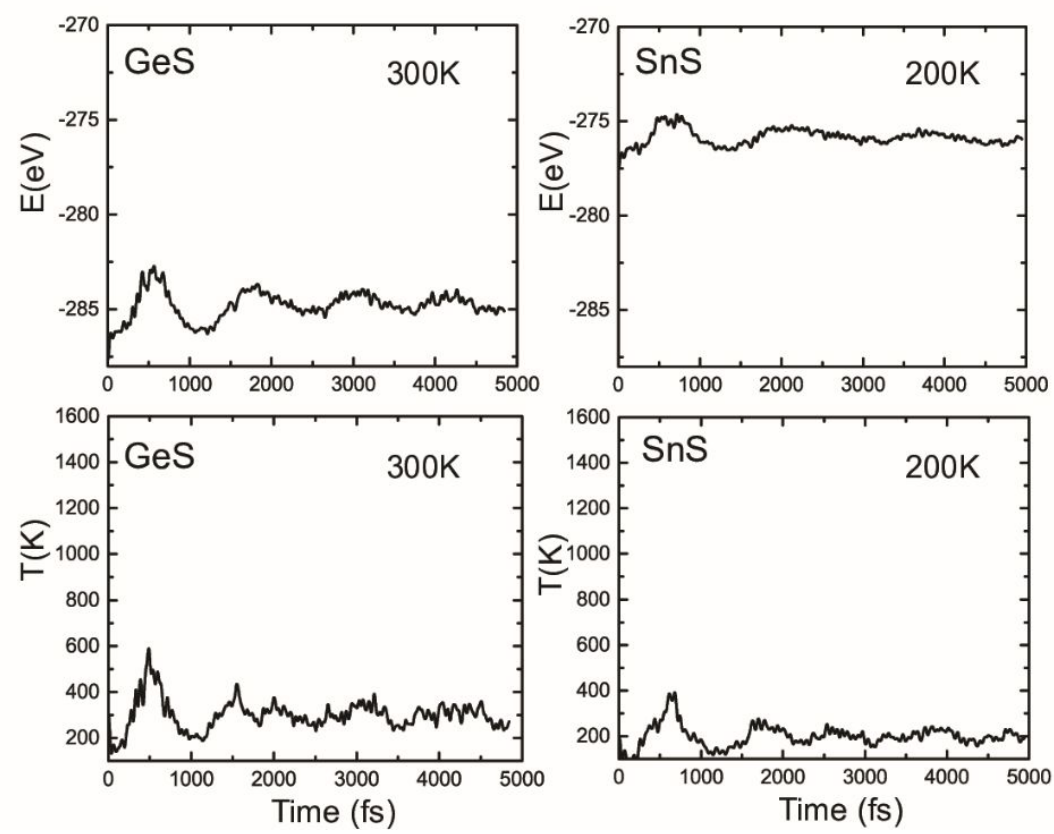

Figure S8. Energy and temperature variations with time for 1D GeS and SnS nanowire.

\section{More details of carrier mobility}

The mobility is estimated by $\mu=\sigma n e,{ }^{8}$ where $\sigma$ indicates the carrier conductivity, $n$ is the carrier density, and $e$ is the elementary charge. To calculate the $\sigma$, a constant carrier relaxation time $\tau$ is estimated using phononlimited scattering model. ${ }^{9}$ The density $n$ can be easily calculated from DFT using the following equations, ${ }^{8}$

$$
\begin{gathered}
n_{0}=e^{-\frac{E_{g}-E_{f}}{k_{B} T}} \int_{E_{g}}^{\infty} d \varepsilon\left[1+e^{-\frac{\varepsilon-E_{f}}{k_{B} T}}\right]^{-1} D(\varepsilon) \text { (for electron density), } \\
p_{0}=e^{-\frac{E_{f}}{k_{B} T}} \int_{-\infty}^{0} d \varepsilon\left[1+e^{-\frac{-\varepsilon}{k_{B} T}}\right]^{-1} D(\varepsilon) \text { (for hole density), }
\end{gathered}
$$

where $D(\varepsilon)$ represents the density of states with zero energy set at the valence band maximum (VBM), $E_{f}$ is the Fermi level, and $E_{\mathrm{g}}$ is band gap. 
Based on Boltzmann transport equations, the anisotropic electrical conductivity $\sigma$ is expressed as following,

$$
\sigma_{i j}(\mu, T)=\frac{e^{2}}{V} \int_{-\infty}^{+\infty} d \varepsilon \sum_{n, k}\left(-\frac{\partial f(\varepsilon, \mu, T)}{\partial \varepsilon}\right) v_{i}(n, k) v_{j}(n, k) \tau(n, k) \delta\left(\varepsilon-E_{n, k}\right)
$$

where $f(\varepsilon, \mu, T)$ is the usual Fermi-Dirac distribution function, defined as

$$
f(\varepsilon, \mu, T)=\frac{1}{e^{(\varepsilon-\mu) / K_{B} T}+1}
$$

$E_{n, k}$ is the energy of the $n$-th band at $k, v(n, k)$ is the $i$ th component of the band velocity at $(n, k)$, and $\tau$ is electron relaxation time. In addition, detailed description of the calculations of electrical conductivity can be found in $P R B$, 68, 125210 (2003) and Comput. Phys. Commun. 2008, 178, 685-699.

To estimate the value of $\tau$, the following equation is used here, ${ }^{10,11}$

$$
\bar{\tau}=\frac{\mathrm{h}^{2} C}{\sqrt{2 \pi k_{B} T}\left|m^{*}\right|^{1 / 2} E_{l}^{2}}
$$

where $C=\left.a_{0}\left(\partial^{2} E / \partial a^{2}\right)\right|_{a=a_{0}}$ the stretching modulus of one-dimensional system, $a_{0}$ is the lattice constant. The carrier effective mass $m^{*}$ is obtained by using the parabolic approximation for the band structure around the band edges, which is expressed as $E_{i}(k)=\mathrm{h}^{2} k^{2} / 2 m_{i}^{*} . E_{l}$ is deformation potential constant of the VBM for hole or the CBM for electron along the transport direction. The detail description of definition can be found in J. Am. Chem. Soc. 131, 17728-17729 (2009) and ACS nano, 5(4): 2593-2600 (2011).

\section{Reference :}

1. Kresse, G.; Furthmüller, J., Efficient iterative schemes for ab initio total-energy calculations using a plane-wave basis set. Phys. Rev. B 1996, 54, 11169. 
2. Kresse, G.; Joubert, D., From ultrasoft pseudopotentials to the projector augmented-wave method. Phys. Rev. B 1999, 59, 1758.

3. King-Smith, R.; Vanderbilt, D., Theory of polarization of crystalline solids. Phys. Rev. B 1993, 47, 1651.

4. Resta, R.; Posternak, M.; Baldereschi, A., Towards a quantum theory of polarization in ferroelectrics: The case of KNbO 3. Phys. Rev. Lett. 1993, 70, 1010.

5. Giannozzi, P.; Baroni, S.; Bonini, N.; Calandra, M.; Car, R.; Cavazzoni, C.; Ceresoli, D.; Chiarotti, G. L.; Cococcioni, M.; Dabo, I.; Corso, A. D.; Gironcoli, S. d.; Fabris, S.; Fratesi, G.; Gebauer, R.; Gerstmann, U.; Gougoussis, C.; Kokalj, A.; Lazzeri, M.; Martin-Samos, L.; Marzari, N.; Mauri, F.; Mazzarello, R.; Paolini, S.; Pasquarello, A.; Paulatto, L.; Sbraccia, C.; Scandolo, S.; Sclauzero, G.; Seitsonen, A. P.; Smogunov, A.; Umari, P.; Wentzcovitch, R. M., QUANTUM ESPRESSO: a modular and open-source software project for quantum simulations of materials. $J$. Phys.: Condens. Matter 2009, 21, 395502.

6. Dong, S.; Yu, R.; Yunoki, S.; Liu, J.-M.; Dagotto, E., Ferromagnetic tendency at the surface of CE-type charge-ordered manganites. Phys. Rev. B 2008, 78, 064414.

7. Dong, S.; Yu, R.; Yunoki, S.; Liu, J.-M.; Dagotto, E., Origin of multiferroic spiral spin order in the R MnO 3 perovskites. Phys. Rev. B 2008, 78, 155121.

8. Yang, J.-H.; Zhang, Y.; Yin, W.-J.; Gong, X.; Yakobson, B. I.; Wei, S.-H., Two-dimensional SiS layers with promising electronic and optoelectronic properties: theoretical prediction. Nano Lett. 2016, 16, 1110-1117.

9. Beleznay, F.; Bogár, F.; Ladik, J., Charge carrier mobility in quasi-one-dimensional systems: Application to a guanine stack. J. Chem. Phys. 2003, 119, 5690-5695.

10. Long, M.-Q.; Tang, L.; Wang, D.; Wang, L.; Shuai, Z., Theoretical predictions of sizedependent carrier mobility and polarity in graphene. J. Am. Chem. Soc. 2009, 131, 17728-17729. 11. Long, M.; Tang, L.; Wang, D.; Li, Y.; Shuai, Z., Electronic structure and carrier mobility in graphdiyne sheet and nanoribbons: theoretical predictions. ACS Nano 2011, 5, 2593-2600. 